\title{
EXISTENCE THEOREM FOR THE FLOW OF AN IDEAL INCOMPRESSIBLE FLUID IN TWO DIMENSIONS*
}

BY

\author{
A. C. SCHAEFFER
}

We shall prove the existence of the flow of an ideal incompressible fluid in two dimensions under certain initial conditions.

Suppose that $R$ is an open connected plane region with a sufficiently smooth boundary. $u(x, y, t)$ and $v(x, y, t)$ are velocity components parallel to the $x$ and $y$ axes respectively and in the positive direction, $p(x, y, t)$ is the pressure, and $\sigma$ is the density.

The flow of an ideal homogeneous incompressible fluid is said to exist if $u(x, y, t), v(x, y, t), p(x, y, t)$ and $\sigma=$ constant are defined in $R$ throughout some time interval, possessing sufficient differentiability and satisfying the conditions:

(1) The divergence is zero and the density is constant.

(2) The normal component of the velocity at the boundary is zero.

(3) The Euler dynamical equations are satisfied

$$
\begin{aligned}
& -\frac{1}{\sigma} \frac{\partial p}{\partial x}=u \frac{\partial u}{\partial x}+v \frac{\partial u}{\partial y}+\frac{\partial u}{\partial t} \\
& -\frac{1}{\sigma} \frac{\partial p}{\partial y}=u \frac{\partial v}{\partial x}+v \frac{\partial v}{\partial y}+\frac{\partial v}{\partial t}
\end{aligned}
$$

It will be proved that if sufficiently smooth velocity components $u(x, y)$ and $v(x, y)$ are defined in $R$ at time $t=0$ such that the divergence is zero and the normal component of the velocity at the boundary is zero, then a flow exists for all time which at time $t=0$ has the given velocity components $u(x, y)$ and $v(x, y)$. That is, $u(x, y, t), v(x, y, t)$, and $p(x, y, t)$ are defined for all time $-\infty<t<\infty$ and for all $x, y$ in $R$ such that at time $t=0$

$$
u(x, y, 0)=u(x, y), \quad v(x, y, 0)=v(x, y) .
$$

In the case of the plane flow of an ideal incompressible fluid the vortex density, $\zeta(x, y, t)=\partial u / \partial y-\partial v / \partial x$, is, according to Helmholtz, constant along the moving point. With initial velocity components $u(x, y)$ and $v(x, y)$ given and satisfying the same conditions as above it is shown that velocity components $u(x, y, t)$ and $v(x, y, t)$, and paths of the moving point $d x / d t=u$,

* Presented to the Society, September 10, 1937; received by the editors September 4, 1936. 
$d y / d t=v, x=a, y=b$ at $t=0$ exist for all time such that conditions (1) and (2) above are satisfied, the vortex density is constant along the path of the moving point, and at time $t=0, u(x, y, 0)=u(x, y)$ and $v(x, y, 0)=v(x, y)$. Then if the region $R$ and the given velocity components have the properties demanded of them in Theorem I, it will follow that $p(x, y, t)$ exists so that the Euler dynamical equations are satisfied. If $R$ is multiply-connected, we have, in order to insure single-valuedness of the pressure, to impose additional conditions (constancy of certain circulations).

Similar results have been obtained by Wolibner and Hölder. For given initial conditions Wolibner* sets up approximating functions which are equicontinuous and, using the selection theorem of Ascoli, shows that a suitable subsequence of the approximating functions converges to a limit defining the flow of an ideal incompressible fluid. Lichtenstein has shown that for given initial conditions the flow of an ideal incompressible fluid exists for a sufficiently small time, and Hölder, $\dagger$ presupposing Lichtenstein's result, shows that the flow in truth exists for all time. Leray, $\ddagger$ by a vastly different method, has obtained an existence theorem for the flow of an incompressible viscous fluid. The novelty of the present paper consists in setting up successive approximations which converge for all time. $\S$

Let $R$ be an open simply-connected\| plane region whose boundary, $B$, consists of a single Jordan curve given in the form $x=x(s), y=y(s)$ where $d^{3} y / d s^{3}$ and $d^{3} x / d s^{3}$ exist and satisfy a uniform Hölder condition of some positive order less than one.

Green's function, $G\left(x, y, x^{\prime}, y^{\prime}\right)$, is defined for all pairs of points $x, y$ and $x^{\prime}, y^{\prime}$ in $R$ by:

$$
G\left(x, y, x^{\prime}, y^{\prime}\right)=\ln \frac{1}{r}+g\left(x, y, x^{\prime}, y^{\prime}\right)
$$

where $r^{2}=\left(x-x^{\prime}\right)^{2}+\left(y-y^{\prime}\right)^{2}$ and, for every point $x, y$ in $R, g\left(x, y, x^{\prime}, y^{\prime}\right)$ considered as a function of $x^{\prime}, y^{\prime}$ is continuous in $R+B$, harmonic in $R$, and has the value $-\ln 1 / r$ on $B$.

THEOREM I. Let velocity components $u(x, y)$ and $v(x, y)$ be defined in $R$ at time $t=0$ such that:

* W. Wolibner, Mathematische Zeitschrift, vol. 37 (1933), pp. 698-726.

$\dagger$ E. Hölder, Mathematische Zeitschrift, vol. 37 (1933), pp. 727-738.

$\ddagger$ J. Leray, Journal de Mathématiques, vol. 12 (1933), pp. 1-82.

$\S$ This paper follows a doctor's thesis submitted at the Massachusetts Institute of Technology and written under the direction of Professor Eberhard Hopf to whom the author wishes to express his gratitude for many valuable suggestions. The thesis contained a proof of uniqueness which is not reproduced here as it has been published elsewhere. See Wolibner, loc. cit.

|| In the appendix multiply-connected regions are considered. 
(a) The partial derivatives of first order of $u$ and $v$ with respect to $x$ and $y$ exist and satisfy a uniform Lipschitz condition,

$$
\left|\frac{\partial u\left(x_{1}, y_{1}\right)}{\partial x}-\frac{\partial u\left(x_{2}, y_{2}\right)}{\partial x}\right| \leqq C\left\{\left(x_{1}-x_{2}\right)^{2}+\left(y_{1}-y_{2}\right)^{2}\right\}^{1 / 2}, \cdots ;
$$

(b) The divergence is zero,

$$
\frac{\partial u}{\partial x}+\frac{\partial v}{\partial y}=0
$$

(c) The normal component of the velocity at the boundary is zero,

$$
u \frac{d y}{d s}-v \frac{d x}{d s}=0
$$

Then there exist velocity components $u(x, y, t)$ and $v(x, y, t)$ defined for all $x, y$ in $R$ and for all time $-\infty<t<\infty$ such that:

(d) $u(x, y, 0)=u(x, y), v(x, y, 0)=v(x, y)$;

(e) The partial derivatives of first order of $u(x, y, t)$ and $v(x, y, t)$ with respect to $x$ and $y$ exist and are continuous;

(f) The divergence is zero;

(g) The normal component of the velocity at the boundary is zero;

(h) The paths of the moving point, $d x / d t=u, d y / d t=v, x=a, y=b$ at $t=0$ exist and at each instant there is a one to one, continuous, area-preserving relation between $(a, b)$ and $(x, y),(a, b)$ in $R+B,(x, y)$ in $R+B$;

(i) The vortex density is constant along the moving point, and in each finite time interval satisfies a uniform Lipschitz condition with respect to $x, y, t$.

Proof. Theorem I is proved by a method of successive approximations. For simplicity we consider a positive time interval $t \geqq 0$, although the same method applies to a negative time interval. Let

$$
\zeta(a, b)=\frac{\partial u(a, b)}{\partial b}-\frac{\partial v(a, b)}{\partial a} .
$$

Then by the hypotheses of Theorem I,

$$
\begin{aligned}
|\zeta(a, b)| & \leqq C_{1}, \\
\left|\zeta\left(a_{1}, b_{1}\right)-\zeta\left(a_{2}, b_{2}\right)\right| & \leqq C_{2}\left\{\left(a_{1}-a_{2}\right)^{2}+\left(b_{1}-b_{2}\right)^{2}\right\}^{1 / 2} .
\end{aligned}
$$

We define lines $x=x_{n}(a, b, t), y=y_{n}(a, b, t), n=0,1,2, \cdots$, issuing from each point $(a, b)$ of the region $R$ at time $t=0$ which will, as $n$ becomes large, approximate the paths of the moving point in the moving fluid. Let

$$
x_{0}(a, b, t) \equiv a, \quad y_{0}(a, b, t) \equiv b .
$$


Suppose that $x_{n-1}(a, b, t)$ and $y_{n-1}(a, b, t)$ have been defined for all time such that at each instant there is a one to one continuous correspondence between $(a, b)$ and $\left(x_{n-1}, y_{n-1}\right),(a, b)$ in $R+B,\left(x_{n-1}, y_{n-1}\right)$ in $R+B$ and such that the first order partial derivatives of $x_{n-1}$ and $y_{n-1}$ with respect to $a$ and $b$ and the first order partial derivatives of $a$ and $b$ with respect to $x_{n-1}$ and $y_{n-1}$ are bounded in every finite time interval. As suggested by the vortex theorem of Helmholtz we define a vortex density which is constant along the lines $x=x_{n-1}, y=y_{n-1}$. Let

$$
\zeta_{n-1}(x, y, t)=\zeta(a, b), \text { where } x=x_{n-1}(a, b, t), \quad y=y_{n-1}(a, b, t) .
$$

Then $\zeta_{n-1}(x, y, t)$ satisfies a Lipschitz condition with respect to $x$ and $y$ in every finite time interval, and for all time has the same bound as $\zeta(a, b)$. Let

$$
\phi_{n}(x, y, t)=-\frac{1}{2 \pi} \int_{R} \zeta_{n-1}\left(x^{\prime}, y^{\prime}, t\right) G\left(x, y, x^{\prime}, y^{\prime}\right) d x^{\prime} d y^{\prime} .
$$

Then $\phi_{n}(x, y, t)$ vanishes on the boundary, and since $\zeta_{n-1}$ satisfies a Lipschitz condition, the second order derivatives of $\phi_{n}$ with respect to $x$ and $y$ exist, are bounded, and satisfy a uniform Hölder condition with respect to $x$ and $y$ in every finite time interval.* Let

$$
u_{n}(x, y, t)=\frac{\partial}{\partial y} \phi_{n}(x, y, t), \quad v_{n}(x, y, t)=-\frac{\partial}{\partial x} \phi_{n}(x, y, t) .
$$

Then the divergence of the velocity components $u_{n}$ and $v_{n}$ is zero and the vortex density is $\zeta_{n-1}$,

Let

$$
\begin{aligned}
& \frac{\partial u_{n}}{\partial x}+\frac{\partial v_{n}}{\partial y}=\frac{\partial^{2} \phi_{n}}{\partial x \partial y}-\frac{\partial^{2} \phi_{n}}{\partial y \partial x}=0 \\
& \frac{\partial u_{n}}{\partial y}-\frac{\partial v_{n}}{\partial x}=\frac{\partial^{2} \phi_{n}}{\partial y^{2}}+\frac{\partial^{2} \phi_{n}}{\partial x^{2}}=\zeta_{n-1}
\end{aligned}
$$

$$
\begin{aligned}
& x_{n}(a, b, t)=a+\int_{0}^{t} u_{n}\left\{x_{n}(a, b, \mu), y_{n}(a, b, \mu), \mu\right\} d \mu, \\
& y_{n}(a, b, t)=b+\int_{0}^{t} v_{n}\left\{x_{n}(a, b, \mu), y_{n}(a, b, \mu), \mu\right\} d \mu .
\end{aligned}
$$

Since $\phi_{n}$ is zero on the boundary and the derivatives of $u_{n}$ and $v_{n}$ with respect to $x$ and $y$ are bounded, it follows that $x_{n}(a, b, t)$ and $y_{n}(a, b, t)$ exist for all time, the point $x_{n}, y_{n}$ remaining in $R+B$, and at each instant there is a one-to-one correspondence between $(a, b)$ and $\left(x_{n}, y_{n}\right),(a, b)$ in $R+B,\left(x_{n}, y_{n}\right)$

* Lichtenstein, Potentialtheorie, Encycklopädie der mathematischen Wissenschaften, vol. 2, part 3, p. 286. 
in $R+B$. Since the derivatives of $u_{n}(x, y, t)$ and $v_{n}(x, y, t)$ with respect to $x$ and $y$ are continuous it follows that $x_{n}(a, b, t)$ and $y_{n}(a, b, t)$ have continuous first order derivatives with respect to $a$ and $b$, and $a$ and $b$ have continuous first order derivatives with respect to $x_{n}$ and $y_{n}$.

$$
\frac{\partial}{\partial a} x_{n}(a, b, t)=1+\int_{0}^{t}\left\{\frac{\partial u_{n}}{\partial x} \frac{\partial x_{n}}{\partial a}+\frac{\partial u_{n}}{\partial y} \frac{\partial y_{n}}{\partial a}\right\} d \mu, \cdots .
$$

Then $x_{n}(a, b, t)$ and $y_{n}(a, b, t)$ have the same properties which $x_{n-1}(a, b, t)$ and $y_{n-1}(a, b, t)$ were supposed to possess, and in addition it is seen that since the divergence is zero the transformation defined by $x_{n}$ and $y_{n}$ is area-preserving.

We thus make the definitions:

$$
x_{0}(a, b, t) \equiv a, \quad y_{0}(a, b, t) \equiv b .
$$

If $x_{n-1}$ and $y_{n-1}$ have been defined, $n \geqq 1$,

$$
\begin{aligned}
\zeta_{n-1}(x, y, t) & =\zeta(a, b), \quad \text { where } \quad x=x_{n-1}(a, b, t), \quad y=y_{n-1}(a, b, t), \\
\phi_{n}(x, y, t) & =-\frac{1}{2 \pi} \int_{R} \zeta_{n-1}\left(x^{\prime}, y^{\prime}, t\right) G\left(x, y, x^{\prime}, y^{\prime}\right) d x^{\prime} d y^{\prime}, \\
u_{n}(x, y, t) & =\frac{\partial}{\partial y} \phi_{n}(x, y, t), \quad v_{n}(x, y, t)=-\frac{\partial}{\partial x} \phi_{n}(x, y, t), \\
x_{n}(a, b, t) & =a+\int_{0}^{t} u_{n}\left\{x_{n}(a, b, \mu), y_{n}(a, b, \mu), \mu\right\} d \mu, \\
y_{n}(a, b, t) & =b+\int_{0}^{t} v_{n}\left\{x_{n}(a, b, \mu), y_{n}(a, b, \mu), \mu\right\} d \mu .
\end{aligned}
$$

The aim now is to show that the approximating functions $u_{n}$ and $v_{n}$ converge as $n$ becomes large to limits which are the velocity components of the moving fluid, and that the approximating lines $x=x_{n}, y=y_{n}$ converge to the paths of the moving points. But before proving convergence it will be shown that the approximating functions have a modulus of continuity which is independent of $n$, and this will follow readily from Lemma I.

LEMMA I. Let $r_{0}$ be the distance between any two points $a_{1}, b_{1}$ and $a_{2}, b_{2}$ at time $t=0$ and $r$ the distance between the corresponding points by the nth mapping function at a later time $t, 0 \leqq t \leqq K$,

$$
\begin{aligned}
& r_{0}^{2}=\left(a_{1}-a_{2}\right)^{2}+\left(b_{1}-b_{2}\right)^{2}, \\
& r^{2}=\left(x_{n}\left(a_{1}, b_{1}, t\right)-x_{n}\left(a_{2}, b_{2}, t\right)\right)^{2}+\left(y_{n}\left(a_{1}, b_{1}, t\right)-y_{n}\left(a_{2}, b_{2}, t\right)\right)^{2} .
\end{aligned}
$$

Then 


$$
r_{0} \leqq C_{3} r, \quad r \leqq C_{3} r_{0}, \quad 0 \leqq t \leqq K,
$$

where $C_{3}$ is independent of $n$, but depends on $K$.

Proof. It is evident from the definition that $\zeta_{n}(x, y, t)$ is bounded independently of $n$ and $t$. Solely from the bound of $\zeta_{n}$, independently of any of its continuity properties, it is found that $r \leqq C r_{0}{ }^{\alpha}, r_{0} \leqq C r^{\alpha}$, where $0<\alpha<1$ and $C$ and $\alpha$ are independent of $n$, and $\alpha$ is a decreasing function of $t$. From this first approximation it follows that $\zeta_{n}(x, y, t)$ does satisfy a uniform Hölder condition in every finite time interval independently of $n$, and from this fact a better approximation can be found.

From definition

$$
\begin{array}{rlrl}
\left|\zeta_{n}(x, y, t)\right| & =|\zeta(a, b)| \leqq C_{1}, & n=0,1,2, \cdots, \\
\phi_{n}(x, y, t) & =-\frac{1}{2 \pi} \int_{R} \zeta_{n-1}\left(x^{\prime}, y^{\prime}, t\right) G\left(x, y, x^{\prime}, y^{\prime}\right) d x^{\prime} d y^{\prime} .
\end{array}
$$

Then if $D_{1} \phi_{n}$ represents the first derivative of $\phi_{n}$ with respect to $x$ or $y$, that is $u_{n}$ or $v_{n}$,

$$
\left|D_{1} \phi_{n}\left(x_{1}, y_{1}, t\right)-D_{1} \phi_{n}\left(x_{2}, y_{2}, t\right)\right| \leqq A_{1} C_{1} r \ln \frac{A_{2}}{r},
$$

where $r^{2}=\left(x_{1}-x_{2}\right)^{2}+\left(y_{1}-y_{2}\right)^{2}$ and the constants $A_{1}$ and $A_{2}$ depend* only on the region $R$.

Let $r_{0}$ and $r$ have the same meaning as in the statement of Lemma I, and let

$$
\begin{array}{ll}
x_{1}=x_{n}\left(a_{1}, b_{1}, t\right), & x_{2}=x_{n}\left(a_{2}, b_{2}, t\right), \\
y_{1}=y_{n}\left(a_{1}, b_{1}, t\right), & y_{2}=y_{n}\left(a_{2}, b_{2}, t\right) .
\end{array}
$$

Then from the definition of $x_{n}$ and $y_{n}$,

$$
\begin{aligned}
x_{1}-x_{2}= & a_{1}-a_{2}+\int_{0}^{t}\left\{u_{n}\left(x_{1}, y_{1}, \mu\right)-u_{n}\left(x_{2}, y_{2}, \mu\right)\right\} d \mu, \\
y_{1}-y_{2}= & b_{1}-b_{2}+\int_{0}^{t}\left\{v_{n}\left(x_{1}, y_{1}, \mu\right)-v_{n}\left(x_{2}, y_{2}, \mu\right)\right\} d \mu, \\
\frac{d r}{d t}= & \frac{x_{1}-x_{2}}{r}\left\{u_{n}\left(x_{1}, y_{1}, t\right)-u_{n}\left(x_{2}, y_{2}, t\right)\right\} \\
& +\frac{y_{1}-y_{2}}{r}\left\{v_{n}\left(x_{1}, y_{1}, t\right)-v_{n}\left(x_{2}, y_{2}, t\right)\right\},
\end{aligned}
$$

* This follows from the fact that $\left|D_{1} G\left(x, y, x^{\prime}, y^{\prime}\right)\right| \leqq C / r$ and $\left|D_{2} G\left(x, y, x^{\prime}, y^{\prime}\right)\right| \leqq C / r^{2}$ where $r^{2}=\left(x-x^{\prime}\right)^{2}+\left(y-y^{\prime}\right)^{2}$ and $C$ depends only on the region $R$. Then $(1 / 2 \pi) \int_{R} \mid D_{1} G\left(x_{1}, y_{1}, x^{\prime}, y^{\prime}\right)$ $-D_{1} G\left(x_{2}, y_{2}, x^{\prime}, y^{\prime}\right) \mid d x^{\prime} d y^{\prime} \leqq A_{1} r \ln A_{2} / r$ where $A_{1}$ and $A_{2}$ depend only on $R$, and $r^{2}=\left(x_{1}-x_{2}\right)^{2}$ $+\left(y_{1}-y_{2}\right)^{2}$. 


$$
\begin{aligned}
&\left|\frac{d r}{d t}\right| \leqq 2 A_{1} C_{1} r \ln \frac{A_{2}}{r}, \\
&\left|\frac{d}{d t} \ln \ln \frac{A_{2}}{r}\right| \leqq 2 A_{1} C_{1}, \\
&\left|\begin{array}{r}
\ln \frac{A_{2}}{r_{0}} \\
\ln \frac{A_{2}}{r} \mid
\end{array}\right| \leqq 2 A_{1} C_{1} t, \\
& r \leqq A_{2} r_{0}^{\exp \left[-2 C_{1} \Lambda_{1} t\right]} \quad r_{0} \leqq A_{2} r^{\exp \left[-2 C_{1} A_{1} t\right]}
\end{aligned}
$$

where it is supposed that $A_{2} \geqq 1$. Then it follows from (5) that $\zeta_{n}(x, y, t)$ satisfies a uniform Hölder condition with respect to $x$ and $y$ in every finite time interval. Suppose that $0 \leqq t \leqq K$ where $K$ is an arbitrary but fixed positive number.

$$
\begin{aligned}
\left|\zeta_{n}\left(x_{1}, y_{1}, t\right)-\zeta_{n}\left(x_{2}, y_{2}, t\right)\right| & =\left|\zeta\left(a_{1}, b_{1}\right)-\zeta\left(a_{2}, b_{2}\right)\right| \\
& \leqq C_{2} r_{0} \leqq C_{2} A_{2} r^{\exp \left[-2 A_{1} C_{1} R\right]}
\end{aligned}
$$

To find a better approximation we consider the smoothness properties of $\phi_{n}(x, y, t)$ in the light of (3) and (6).

$$
\begin{gathered}
\phi_{n}(x, y, t)=-\frac{1}{2 \pi} \int_{R} \zeta_{n-1}\left(x^{\prime}, y^{\prime}, t\right) G\left(x, y, x^{\prime}, y^{\prime}\right) d x^{\prime} d y^{\prime} . \\
\left\{\begin{array}{lc}
\left|D_{1} \phi_{n}(x, y, t)\right| \leqq A_{3} C_{1}, & 0 \leqq t<\infty, \\
\left|D_{2} \phi_{n}(x, y, t)\right| \leqq C_{4}\left(C_{1}+C_{2} A_{2}\right), & 0 \leqq t \leqq K, \\
\left|D_{2} \phi_{n}\left(x_{1}, y_{1}, t\right)-D_{2} \phi_{n}\left(x_{2}, y_{2}, t\right)\right| \leqq C_{5}\left(C_{1}+C_{2} A_{2}\right) r^{\exp \left[-2 A_{1} C_{1} K\right]}, & 0 \leqq t \leqq K,
\end{array}\right.
\end{gathered}
$$

where $r^{2}=\left(x_{1}-x_{2}\right)^{2}+\left(y_{1}-y_{2}\right)^{2}$ and the constant $A_{3}$ depends* only on the region $R$, and $C_{4}$ and $C_{5}$ depend on the region $R$ and on $K$, but are independent of $n$.

From the inequalities (7) it can be inferred that the first derivatives of $x_{n}$ and $y_{n}$ are bounded independently of $n$. First, since the derivatives of $u_{n}(x, y, t)$ and $v_{n}(x, y, t)$ with respect to $x$ and $y$ are continuous in $x$ and $y$ and bounded in each finite time interval, differentiation under the integral sign by $a$ or $b$ is justified.

$$
\frac{\partial}{\partial a} x_{n}(a, b, t)=1+\int_{0}^{t}\left\{\frac{\partial u_{n}}{\partial x} \frac{\partial x_{n}}{\partial a}+\frac{\partial u_{n}}{\partial y} \frac{\partial y_{n}}{\partial a}\right\} d \mu, \cdots .
$$

* Lichtenstein, loc. cit. 
Then from the bound on the derivatives of $u_{n}$ and $v_{n}$ one finds:

$$
\left|\frac{\partial x_{n}}{\partial a}\right|,\left|\frac{\partial x_{n}}{\partial b}\right|,\left|\frac{\partial y_{n}}{\partial a}\right|,\left|\frac{\partial y_{n}}{\partial b}\right| \leqq e^{2 C_{4}\left(C_{1}+C_{2} A_{2}\right) t}, \quad 0 \leqq t \leqq K
$$

and in the same way

$$
\left|\frac{\partial a}{\partial x_{n}}\right|,\left|\frac{\partial a}{\partial y_{n}}\right|,\left|\frac{\partial b}{\partial x_{n}}\right|,\left|\frac{\partial b}{\partial y_{n}}\right| \leqq e^{2 C_{4}\left(C_{1}+C_{2} A_{2}\right) t}, \quad 0 \leqq t \leqq K .
$$

Lemma I follows from this since the bound $e^{2 C_{1}\left(C_{1}+C_{2} \Lambda_{2}\right) K}$ is independent of $n$.

Note that another approximation shows that $\zeta_{n}(x, y, t)$ satisfies a Lipschitz condition which is independent of $n$ but depends on the time. Letting $\left(x_{1}, y_{1}\right)$ and $\left(x_{2}, y_{2}\right)$ be two points at time $t$ a distance $r$ apart and $\left(a_{1}, b_{1}\right)$ and $\left(a_{2}, b_{2}\right)$ the corresponding points by the $n$th mapping function at time $t=0$ a distance $r_{0}$ apart we have from Lemma I

$$
\left|\zeta_{n}\left(x_{1}, y_{1}, t\right)-\zeta_{n}\left(x_{2}, y_{2}, t\right)\right|=\left|\zeta\left(a_{1}, b_{1}\right)-\zeta\left(a_{2}, b_{2}\right)\right| \leqq C_{2} r_{0} \leqq C_{2} C_{3} r,
$$

where $r^{2}=\left(x_{1}-x_{2}\right)^{2}+\left(y_{1}-y_{2}\right)^{2}$. From (7)

(9) $\begin{cases}\left|u_{n}(x, y, t)\right|, \quad\left|v_{n}(x, y, t)\right| \leqq A_{3} C_{1}, & 0 \leqq t<\infty, \\ \left|u_{n}\left(x_{1}, y_{1}, t\right)-u_{n}\left(x_{2}, y_{2}, t\right)\right| \leqq C_{6}\left(\left|x_{1}-x_{2}\right|+\left|y_{1}-y_{2}\right|\right), & 0 \leqq t \leqq K, \\ \left|v_{n}\left(x_{1}, y_{1}, t\right)-v_{n}\left(x_{2}, y_{2}, t\right)\right| \leqq C_{6}\left(\left|x_{1}-x_{2}\right|+\left|y_{1}-y_{2}\right|\right), & 0 \leqq t \leqq K,\end{cases}$

where $A_{3} C_{1}$ is independent of $n$ and $t$, and $C_{6}$ depends on $K$ but not on $n$.

It can now be shown that the approximating functions $\zeta_{n}, u_{n}, v_{n}, x_{n}$, and $y_{n}$ converge as $n$ becomes large to limits which have the properties stated in Theorem I. Choosing any fixed positive number $K$ consider the time interval $0 \leqq t \leqq K$. From the boundedness of $\zeta_{n}(x, y, t)$,

$$
\left|\zeta_{1}(x, y, t)-\zeta_{0}(x, y, t)\right| \leqq 2 C_{1}, \quad 0 \leqq t<\infty .
$$

Suppose it has been proved that

$$
\left|\zeta_{n}(x, y, t)-\zeta_{n-1}(x, y, t)\right| \leqq 2 C_{1} \frac{(m t)^{n-1}}{(n-1) !}, \quad 0 \leqq t \leqq K .
$$

Then since $\int_{R}\left|D_{1} G\left(x, y, x^{\prime}, y^{\prime}\right)\right| d x^{\prime} d y^{\prime}$ is uniformly bounded in $R$ one obtains from the definition of $u_{n}$ and $v_{n}$,

$$
\begin{array}{cl}
\left|u_{n+1}(x, y, t)-u_{n}(x, y, t)\right| \leqq 2 C_{1} C_{7} \frac{(m t)^{n-1}}{(n-1) !}, & 0 \leqq t \leqq K, \\
\left|v_{n+1}(x, y, t)-v_{n}(x, y, t)\right| \leqq 2 C_{1} C_{7} \frac{(m t)^{n-1}}{(n-1) !}, & 0 \leqq t \leqq K,
\end{array}
$$


where $C_{7}$ depends only on the region $R$. From the inequality (10) we can find a bound on the distance between the lines $x=x_{n}, y=y_{n}$ and $x=x_{n+1}, y=y_{n+1}$ issuing from the same point $(a, b)$ at time $t=0$ (the inequality (11)), and since the relation is defined by an integral the exponent $n-1$ is raised to $n$.

$$
\begin{aligned}
x_{n+1}(a, b, t)-x_{n}(a, b, t)= & \int_{0}^{t}\left\{u_{n+1}\left(x_{n+1}, y_{n+1}, \mu\right)-u_{n}\left(x_{n+1}, y_{n+1}, \mu\right)\right\} d \mu \\
& +\int_{0}^{t}\left\{u_{n}\left(x_{n+1}, y_{n+1}, \mu\right)-u_{n}\left(x_{n}, y_{n}, \mu\right)\right\} d \mu
\end{aligned}
$$

and a similar expression for $y_{n+1}-y_{n}$. Applying (10) to the first term on the right and (9) to the second term on the right and adding the resulting inequalities one obtains

$$
\begin{aligned}
& \left|x_{n+1}-x_{n}\right|+\left|y_{n+1}-y_{n}\right| \\
& \quad \leqq 4 C_{1} C_{7} m^{n-1} \frac{t^{n}}{n !}+2 C_{6} \int_{0}^{t}\left\{\left|x_{n+1}-x_{n}\right|+\left|y_{n+1}-y_{n}\right|\right\} d \mu,
\end{aligned}
$$

where on the left the four variables are taken at time $t$, and on the right they are taken at time $\mu$. To show that this implies (11) suppose it has been shown that the left-hand side of this inequality is less than $4 C_{1} C_{7} m^{n-1}\left(t^{n} / n !\right) e^{2 C_{6} t}$ $+A\left(2 C_{6} t\right)^{p} / p$ ! in the time interval $0 \leqq t \leqq K$, where $A$ is a positive constant and $p$ is an integer or zero. On putting this in the right and performing the indicated integration it is found that the left-hand side is less than $4 C_{1} C_{7} m^{n-1}\left(t^{n} / n !\right) e^{2 C_{6} t}+A\left(2 C_{6} t\right)^{p+1} /(p+1)$ !. The constant $A$ can be made so large that this is true for the case $p=0$, and then it follows for all $p$. Thus

$$
\begin{aligned}
\left|x_{n+1}(a, b, t)-x_{n}(a, b, t)\right|+\left|y_{n+1}(a, b, t)-y_{n}(a, b, t)\right| & \\
& \leqq 4 C_{1} C_{7} m^{n-1} \frac{t^{n}}{n !} e^{2 C_{6} t} .
\end{aligned}
$$

If a point $(a, b)$ at time $t=0$ is considered, (11) gives a bound on the distance between the corresponding points $\left(x_{n}, y_{n}\right)$ and $\left(x_{n+1}, y_{n+1}\right)$ by the $n$ and $n+1$ mapping functions. If a point $(x, y)$ at time $t>0$ is considered, there correspond two points at time $t=0,\left(a_{1}, b_{1}\right)$ and $\left(a_{2}, b_{2}\right)$ by the $n$ and $n+1$ mapping functions respectively. By the use of Lemma $I$, the inequality (11) can be transformed into a bound on the distance between $\left(a_{1}, b_{1}\right)$ and $\left(a_{2}, b_{2}\right)$. Let

$$
\begin{aligned}
& x=x_{n}\left(a_{1}, b_{1}, t\right)=x_{n+1}\left(a_{2}, b_{2}, t\right), \\
& y=y_{n}\left(a_{1}, b_{1}, t\right)=y_{n+1}\left(a_{2}, b_{2}, t\right) .
\end{aligned}
$$

We introduce the auxiliary point $x_{n}\left(a_{2}, b_{2}, t\right), y_{n}\left(a_{2}, b_{2}, t\right)$ and use Lemma I. 


$$
\begin{aligned}
\left|a_{1}-a_{2}\right|+\left|b_{1}-b_{2}\right| \leqq & 2 C_{3}\left\{\left|x_{n}\left(a_{1}, b_{1}, t\right)-x_{n}\left(a_{2}, b_{2}, t\right)\right|\right. \\
& \left.+\left|y_{n}\left(a_{1}, b_{1}, t\right)-y_{n}\left(a_{2}, b_{2}, t\right)\right|\right\} \\
= & 2 C_{3}\left\{\left|x_{n+1}\left(a_{2}, b_{2}, t\right)-x_{n}\left(a_{2}, b_{2}, t\right)\right|\right. \\
& \left.+\left|y_{n+1}\left(a_{2}, b_{2}, t\right)-y_{n}\left(a_{2}, b_{2}, t\right)\right|\right\},
\end{aligned}
$$

which by (11) is less than $8 C_{1} C_{3} C_{7} m^{n-1}\left(t^{n} / n !\right) e^{2 C_{6} t}, 0 \leqq t \leqq K$. By construction, $\zeta_{n}(x, y, t)=\zeta\left(a_{1}, b_{1}\right)$ and $\zeta_{n+1}(x, y, t)=\zeta\left(a_{2}, b_{2}\right)$, so using (2) and the last found inequality we have

$$
\begin{aligned}
\left|\zeta_{n+1}(x, y, t)-\zeta_{n}(x, y, t)\right| & =\left|\zeta\left(a_{2}, b_{2}\right)-\zeta\left(a_{1}, b_{1}\right)\right| \\
\leqq & C_{2}\left\{\left|a_{1}-a_{2}\right|+\left|b_{1}-b_{2}\right|\right\} \leqq 8 C_{1} C_{2} C_{3} C_{7} m^{n-1} \frac{t^{n}}{n !} e^{2 C_{6} t}
\end{aligned}
$$

The coefficient $4 C_{2} C_{3} C_{7} e^{2 C_{6} K}$ is independent of $n$, but depends on $K$; so, letting $m$ be as large as this coefficient, we have

$$
\left|\zeta_{n+1}(x, y, t)-\zeta_{n}(x, y, t)\right| \leqq 2 C_{1} \frac{(m t)^{n}}{n !}, \quad 0 \leqq t \leqq K .
$$

This inequality then holds for all $n$, where $C_{1}$ and $m$ are independent of $n$, but $m$ depends on $K$. This also verifies the inequalities (10) and (11) so the functions $\zeta_{n}(x, y, t), u_{n}(x, y, t), v_{n}(x, y, t), x_{n}(a, b, t)$, and $y_{n}(a, b, t)$ converge to continuous limits and uniformly in every finite time interval. Dropping the subscripts in the limits it follows from (8) that $\zeta(x, y, t)$ satisfies a Lipschitz condition with respect to $x$ and $y$ in every finite time interval.

$$
\left|\zeta\left(x_{1}, y_{1}, t\right)-\zeta\left(x_{2}, y_{2}, t\right)\right| \leqq C_{2} C_{3}\left\{\left(x_{1}-x_{2}\right)^{2}+\left(y_{1}-y_{2}\right)^{2}\right\}^{1 / 2}, \quad 0 \leqq t \leqq K,
$$

where $C_{2}$ is a given constant and $C_{3}$ depends on $K$.

The relations between the variables are preserved in the limits.

$$
\begin{array}{rlrl}
\phi(x, y, t)= & -\frac{1}{2 \pi} \int_{R} \zeta\left(x^{\prime}, y^{\prime}, t\right) G\left(x, y, x^{\prime}, y^{\prime}\right) d x^{\prime} d y^{\prime}, & \\
u(x, y, t)= & \frac{\partial}{\partial y} \phi(x, y, t), & u=\operatorname{limit} u_{n} \\
v(x, y, t)= & -\frac{\partial}{\partial x} \phi(x, y, t), & v=\text { limit } v_{n} \\
D_{2} \phi= & -\frac{\zeta(x, y, t)}{2 \pi} D_{2}\left\{\int_{R} G\left(x, y, x^{\prime}, y^{\prime}\right) d x^{\prime} d y^{\prime}\right\} & \\
& -\frac{1}{2 \pi} \int_{R}\left\{\zeta\left(x^{\prime}, y^{\prime}, t\right)-\zeta(x, y, t)\right\} D_{2} G\left(x, y, x^{\prime}, y^{\prime}\right) d x^{\prime} d y^{\prime} .
\end{array}
$$


From this and similar expressions for $D_{2} \phi_{n}$ it follows that the first derivatives of $u_{n}(x, y, t)$ and $v_{n}(x, y, t)$ with respect to $x$ and $y$ converge to the corresponding derivatives of $u(x, y, t)$ and $v(x, y, t)$. The divergence is zero and $\zeta(x, y, t)$ is the vortex density of the moving fluid.

$$
\begin{aligned}
& \frac{\partial}{\partial x} u(x, y, t)+\frac{\partial}{\partial y} v(x, y, t)=\frac{\partial^{2}}{\partial x \partial y} \phi(x, y, t)-\frac{\partial^{2}}{\partial y \partial x} \phi(x, y, t)=0, \\
& \frac{\partial}{\partial y} u(x, y, t)-\frac{\partial}{\partial x} v(x, y, t)=\frac{\partial^{2}}{\partial y^{2}} \phi(x, y, t)+\frac{\partial^{2}}{\partial x^{2}} \phi(x, y, t)=\zeta(x, y, t) .
\end{aligned}
$$

From the uniform convergence of $x_{n}, y_{n}, u_{n}$, and $v_{n}$ to their respective limits and the equicontinuity of $u_{n}$ and $v_{n}$ expressed in (9) it follows that

$$
\begin{aligned}
& x(a, b, t)=a+\int_{0}^{t} u(x(a, b, \mu), y(a, b, \mu), \mu) d \mu, \quad x=\text { limit } x_{n}, \\
& y(a, b, t)=b+\int_{0}^{t} v(x(a, b, \mu), y(a, b, \mu), \mu) d \mu, \quad y=\text { limit } y_{n} .
\end{aligned}
$$

At each instant there is a one-to-one continuous correspondence between $(a, b)$ and $(x(a, b, t), y(a, b, t)),(a, b)$ in $R+B,(x, y)$ in $R+B$, and since the divergence is zero the transformation is area-preserving.

The vortex density is constant along the moving point, $\zeta(x, y, t)=\zeta(a, b)$, where $x=x(a, b, t), y=y(a, b, t)$.

Since $\zeta(x, y, 0)=\zeta(x, y)=\partial u(x, y) / \partial y-\partial v(x, y) / \partial x$, where $u(x, y)$ and $v(x, y)$ are the given velocity components and since the divergence is zero,

$$
u(x, y, 0)=u(x, y), \quad v(x, y, 0)=v(x, y) .
$$

These functions have all the properties stated in the conclusions of Theorem $I$, except that it remains to show that $\zeta(x, y, t)$ satisfies a Lipschitz condition with respect to the time. Let $t_{1}$.be greater than $t_{2}$. To study the difference $\zeta\left(x, y, t_{1}\right)-\zeta\left(x, y, t_{2}\right)$ consider the moving point which passes through the point $(x, y)$ at time $t_{1}$. Its coordinates, $\left(x^{\prime}, y^{\prime}\right)$, at time $t_{2}$ satisfy the relations

$$
\begin{aligned}
& x=x^{\prime}+\int_{t_{2}}^{t_{1}} u d \mu, \\
& y=y^{\prime}+\int_{t_{2}}^{t_{1}} v d \mu,
\end{aligned}
$$

and since, by (9), $u$ and $v$ are bounded we have

$$
\left|x-x^{\prime}\right|+\left|y-y^{\prime}\right| \leqq 2 A_{3} C_{1}\left|t_{1}-t_{2}\right| \text {. }
$$

Then since $\zeta$ is constant along the moving point, we have from (8) 


$$
\begin{aligned}
\left|\zeta\left(x, y, t_{1}\right)-\zeta\left(x, y, t_{2}\right)\right| & =\left|\zeta\left(x^{\prime}, y^{\prime}, t_{2}\right)-\zeta\left(x, y, t_{2}\right)\right| \\
& \leqq C_{2} C_{3}\left\{\left|x-x^{\prime}\right|+\left|y-y^{\prime}\right|\right\} \leqq 2 A_{3} C_{1} C_{2} C_{3}\left|t_{1}-t_{2}\right|,
\end{aligned}
$$

where the constant $2 A_{3} C_{1} C_{2} C_{3}$ depends on $K, 0 \leqq t \leqq K$. It follows from this that the first order derivatives of $u(x, y, t)$ and $v(x, y, t)$ are continuous in $x, y, t$; and that $u(x, y, t)$ and $v(x, y, t)$ satisfy a Lipschitz condition with respect to $x, y, t$. This completes the proof of Theorem I.

From Theorem I it can be inferred that the velocity components $u(x, y, t)$ and $v(x, y, t)$ are differentiable with respect to the time. To show this we first outline the proof that $\partial \phi(x, y, t) / \partial t$ exists, $\phi$ being the flow function.

$$
\phi(x, y, t)=-\frac{1}{2 \pi} \int_{R} \zeta\left(x^{\prime}, y^{\prime}, t\right) G\left(x, y, x^{\prime}, y^{\prime}\right) d x^{\prime} d y^{\prime}=-\frac{1}{2 \pi} \int_{R_{\mathrm{o}}}-\frac{1}{2 \pi} \int_{R-R_{\mathrm{o}}},
$$

where $R_{0}$ is a circle of radius $\epsilon>0$ with center at $x, y$. The paths of the moving point issuing from points $(a, b)$ of the region $R$ at time $t=0$ and passing through points $\left(x^{\prime}, y^{\prime}\right)$ at time $t$ define a one-to-one, continuous, area-preserving relation between $(a, b)$ and $\left(x^{\prime}, y^{\prime}\right), x^{\prime}=x^{\prime}(a, b, t), y^{\prime}=y^{\prime}(a, b, t)$. The points $\left(x^{\prime}, y^{\prime}\right)$ lying in $R-R_{0}$ correspond to points $(a, b)$ lying in a region which we call $R-R_{1}$. In the integral over the region $R-R_{0}$ at time $t$ we transform the variables of integration from $x^{\prime}, y^{\prime}$ to $a, b$, the region of integration becoming $R-R_{1}$, and since the vortex density is constant along the moving point we have

$$
\begin{aligned}
& -\frac{1}{2 \pi} \int_{R-R_{0}} \zeta\left(x^{\prime}, y^{\prime}, t\right) G\left(x, y, x^{\prime}, y^{\prime}\right) d x^{\prime} d y^{\prime} \\
= & -\frac{1}{2 \pi} \int_{R-R_{1}} \zeta(a, b, 0) G\left(x, y, x^{\prime}, y^{\prime}\right) d a d b,
\end{aligned}
$$

where in $G\left(x, y, x^{\prime}, y^{\prime}\right)$ on the right $x^{\prime}=x^{\prime}(a, b, t), y^{\prime}=y^{\prime}(a, b, t)$. In the integral over the region $R_{0}$ we substitute the letters $a, b$ for $x^{\prime}, y^{\prime}$, this being a change in nomenclature and not a transformation of variables. By definition

$$
\phi(x, y, 0)=-\frac{1}{2 \pi} \int_{R} \zeta(a, b, 0) G(x, y, a, b) d a d b .
$$

Thus one obtains

$$
\begin{gathered}
\frac{\phi(x, y, t)-\phi(x, y, 0)}{t}=-\frac{1}{2 \pi} \int_{R_{0}}\left\{\frac{\zeta(a, b, t)-\zeta(a, b, 0)}{t}\right\} G(x, y, a, b) d a d b \\
-\frac{1}{2 \pi} \int_{R-R_{0}} \zeta(a, b, 0)\left\{\frac{G\left(x, y, x^{\prime}, y^{\prime}\right)-G(x, y, a, b)}{t}\right\} d a d b
\end{gathered}
$$




$$
\begin{aligned}
& +\left\{-\frac{1}{2 \pi t} \int_{R_{0}-R_{1}}+\frac{1}{2 \pi t} \int_{R_{1}-R_{0}}\right\}\left\{\zeta(a, b, 0) G\left(x, y, x^{\prime}, y^{\prime}\right) d a d b\right\} \\
= & \mathrm{I}+\mathrm{II}+\mathrm{III}+\mathrm{IV} .
\end{aligned}
$$

In the expression II,

$$
\frac{x^{\prime}-a}{t} \rightarrow u(a, b, 0), \quad \frac{y^{\prime}-b}{t} \rightarrow v(a, b, 0) \quad \text { as } \quad t \rightarrow 0 .
$$

By choosing first $\epsilon$ small and then $t$ small it can be shown that I, III, and IV approach zero and II approaches a finite limit.

$$
\begin{aligned}
& \lim _{t \rightarrow 0} \frac{\phi(x, y, t)-\phi(x, y, 0)}{t} \\
& \quad=-\frac{1}{2 \pi} \int_{R} \zeta(a, b, 0)\left\{u(a, b, 0) \frac{\partial G(x, y, a, b)}{\partial a}+v(a, b, 0) \frac{\partial G(x, y, a, b)}{\partial b}\right\} d a d b .
\end{aligned}
$$

This shows that $\partial \phi / \partial t$ exists at time $t=0$, and in the same way it exists for all time. Since $\zeta, u$, and $v$ satisfy a Lipschitz condition with respect to $x, y, t$, it is well known in potential theory that the first order derivatives of $\partial \phi / \partial t$ with respect to $x$ and $y$ exist and are continuous in $x, y, t$. Then the order of differentiation is immaterial,

$$
\frac{\partial}{\partial y} \frac{\partial \phi}{\partial t}=\frac{\partial}{\partial t} \frac{\partial \phi}{\partial y} \equiv \frac{\partial u}{\partial t}
$$

so that $\partial u / \partial t$ and $\partial v / \partial t$ exist and are continuous.

To construct the pressure, let $C$ be any closed Jordan curve with continuously turning tangent moving with the fluid. If $R_{C}$ is the interior of $C$ we have

$$
-\int_{R_{C}} \zeta(x, y, t) d x d y=\int_{C} u d x+v d y
$$

and since $\zeta$ is constant along the moving point and the fluid is incompressible $\int_{R_{C}} \zeta d x d y$ is a constant independent of the time,

$$
\int_{C} u d x+v d y=\text { constant. }
$$

Differentiating along the moving curve we have

$$
\begin{aligned}
\int_{C}\left\{\left(u \frac{\partial u}{\partial x}+v \frac{\partial u}{\partial y}+\frac{\partial u}{\partial t}\right) d x\right. & \left.+\left(u \frac{\partial v}{\partial x}+v \frac{\partial v}{\partial y}+\frac{\partial v}{\partial t}\right) d y\right\} \\
& +\frac{1}{2} \int_{C} \frac{\partial}{\partial s}\left(u^{2}+v^{2}\right) d s=0 .
\end{aligned}
$$


The second integral is zero around any closed curve so the first is also. Thus a pressure defined by

$$
p(x, y, t)=-\sigma \int_{x_{0}, y_{0}}^{x, y}\left\{\left(u \frac{\partial u}{\partial x}+v \frac{\partial u}{\partial y}+\frac{\partial u}{\partial t}\right) d x+\left(u \frac{\partial v}{\partial x}+v \frac{\partial v}{\partial y}+\frac{\partial v}{\partial t}\right) d y\right\}
$$

exists and satisfies the Euler dynamical equations.

\section{APPENDIX}

It was assumed in Theorem I that the plane region $R$ is simply connected, but this is an unnecessary restriction. Theorem I may be extended so as to apply to multiply-connected regions, but we shall consider only the extension to doubly-connected regions as this will illustrate the principle.

Let $R$ be an open doubly-connected plane region whose boundary, $B$, consists of two distinct Jordan curves such that if $x=x(s), y=y(s)$ defines either of the curves, $d^{3} x / d s^{3}$ and $d^{3} y / d s^{3}$ exist and satisfy a uniform Hölder condition of some positive order less than one.

In Theorem I the same hypotheses (a), (b), and (c) are made and all the conclusions (d), (e), (f), (g), (h), and (i) are drawn with the additional statement (j).

(j) The circulation around the inner boundary is a given constant independent of the time.

The constancy of this circulation will insure the existence of a single-valued pressure.

In the proof the following definitions are made:

$$
x_{0}(a, b, t) \equiv a, \quad y_{0}(a, b, t) \equiv b .
$$

If $x_{n-1}(a, b, t)$ and $y_{n-1}(a, b, t)$ have been defined, $n \geqq 1$,

$$
\begin{aligned}
\zeta_{n-1}(x, y, t) & =\zeta(a, b), \quad \text { where } \quad x=x_{n-1}(a, b, t), \quad y=y_{n-1}(a, b, t), \\
\phi_{n}(x, y, t) & =-\frac{1}{2 \pi} \int_{R} \zeta_{n-1}\left(x^{\prime}, y^{\prime}, t\right) G\left(x, y, x^{\prime}, y^{\prime}\right) d x^{\prime} d y^{\prime}+f_{n}(t) h(x, y), \\
u_{n}(x, y, t) & =\frac{\partial}{\partial y} \phi_{n}(x, y, t), \quad v_{n}(x, y, t)=-\frac{\partial}{\partial x} \phi_{n}(x, y, t), \\
x_{n}(a, b, t) & =a+\int_{0}^{t} u_{n}\left(x_{n}(a, b, \mu), y_{n}(a, b, \mu), \mu\right) d \mu \\
y_{n}(a, b, t) & =b+\int_{0}^{t} v_{n}\left(x_{n}(a, b, \mu), y_{n}(a, b, \mu), \mu\right) d \mu .
\end{aligned}
$$

Let $h(x, y)$ be continuous in $R+B$, harmonic in $R$, have the value one on 
the inner boundary, and vanish on the outer boundary of $R . f_{n}(t)$ is a function to be defined presently.

It is seen that the only variation from the case of a simply connected region is that here the function $f_{n}(t) h(x, y)$ enters. At time $t=0$ the circulation around $q_{1}$ ( $q_{1}$ is the inner boundary, and $q_{2}$ is the outer boundary of $R$ ) due to the velocity components $u(x, y)$ and $v(x, y)$ is a given constant determined by these given velocity components. Suppose the value of this circulation is $W$. We shall define $f_{n}(t)$ so that the circulation around $q_{1}$ due to the velocity components $u_{n}(x, y, t)$ and $v_{n}(x, y, t)$ is for all time the given constant $W$.

From definition of $u_{n}$ and $v_{n}$ the circulation around $q_{1}$ is

$$
\int_{q_{1}} u_{n} d x+v_{n} d y=-\int_{q_{1}} \frac{\partial \phi_{n}}{\partial \nu} d s
$$

where $\nu$ is the direction of the inner normal. The right-hand side of this equation may be transformed by means of Green's second identity

$$
\int_{R} h(x, y) \zeta_{n-1}(x, y, t) d x d y=-\int_{q_{1}} \frac{\partial \phi_{n}}{\partial \nu} d s+f_{n}(t) \int_{q_{1}} \frac{\partial h}{\partial \nu} d s
$$

where $h$ is the harmonic function defined above. Defining $f_{n}(t)$ by

$$
f_{n}(t)=\frac{1}{\lambda}\left\{-W+\int_{R} h(x, y) \zeta_{n-1}(x, y, t) d x d y\right\},
$$

where $\lambda=\int_{q_{1}}(\partial h / \partial \nu) d s \neq 0$, it follows that the circulation around $q_{1}$ due to the velocity components $u_{n}$ and $v_{n}$ is for all time the given constant $W$.

The proof of Theorem $I$ is not essentially altered by the introduction of the function $f_{n}(t) h(x, y)$ in the definition of $\phi_{n}(x, y, t)$. First, from constructon it is seen that $\zeta_{n}(x, y, t)$ is uniformly bounded, and by (12) this implies that $f_{n}(t)$ is uniformly bounded,

$$
\left|f_{n}(t)\right| \leqq\left|\frac{1}{\lambda}\right|\left\{|W|+C_{1} \int_{R} d x d y\right\} \leqq C_{8},
$$

where $C_{8}$ is independent of $n, x, y, t$. Due to the smoothness properties of the boundary of $R$ the harmonic function $h(x, y)$ has continuous* (and bounded) third order derivatives in $R+B$, so the third order partial derivatives of $f_{n}(t) h(x, y)$ with respect to $x$ and $y$ are bounded independently of $n, x, y, t$.

Lemma $I$ is proved as before. The inequality (4) is true with a larger constant in place of $A_{1} C_{1}$,

$$
\left|D_{1} \phi_{n}\left(x_{1}, y_{1}, t\right)-D_{1} \phi_{n}\left(x_{2}, y_{2}, t\right)\right| \leqq C_{9} r \ln \frac{A_{2}}{r},
$$

\footnotetext{
* Kellogg, these Transactions, vol. 9 (1908), pp. 39-50 and vol. 13 (1912), pp. 108-132.
} 
where $C_{9}$ is independent of $n, x, y, t$. From this one derives a first approximation, the inequality (5) with $A_{1} C_{1}$ replaced by $C_{9}$. Then using this result another approximation proves Lemma I, and the inequalities (8) and (9) with constants independent of $n$ also follow.

To show that the sequence $\zeta_{n}(x, y, t)$ converges we have first from the boundedness of $\zeta_{n}$,

$$
\left|\zeta_{1}(x, y, t)-\zeta_{0}(x, y, t)\right| \leqq 2 C_{1}, \quad 0 \leqq t<\infty .
$$

Suppose it has been shown that

$$
\left|\zeta_{n}(x, y, t)-\zeta_{n-1}(x, y, t)\right| \leqq 2 C_{1} \frac{(m t)^{n-1}}{(n-1) !}, \quad 0 \leqq t \leqq K .
$$

Then from (12) it follows that

$$
\left|f_{n+1}(t)-f_{n}(t)\right| \leqq\left|\frac{1}{\lambda}\right| \int_{R}\left|\zeta_{n}(x, y, t)-\zeta_{n-1}(x, y, t)\right| d x d y,
$$

and finally

$$
\left|u_{n+1}(x, y, t)-u_{n}(x, y, t)\right| \leqq 2 C_{1} C_{10} \frac{(m t)^{n-1}}{(n-1) !}
$$

with a similar inequality for $v_{n+1}-v_{n}$, where $C_{10}$ is independent of $n, x, y, t$. The same procedure that was used in the proof of Theorem I shows that

$$
\left|\zeta_{n+1}(x, y, t)-\zeta_{n}(x, y, t)\right| \leqq 2 C_{1} \frac{(m t)^{n}}{n:}, \quad 0 \leqq t \leqq K,
$$

if $m$ is a sufficiently large constant which depends on $K$ but not on $n$.

This implies that $\zeta_{n}, u_{n}, v_{n}, x_{n}, y_{n}$ and now, by (13), $f_{n}(t)$ converge uniformly in every finite time interval. By uniform convergence the relation (12) holds in the limits

$$
f(t)=\frac{1}{\lambda}\left\{-W+\int_{R} \zeta(x, y, t) h(x, y) d x d y\right\},
$$

where $f(t)=\operatorname{limit} f_{n}(t), \zeta=\operatorname{limit} \zeta_{n}$. Then the circulation around $q_{1}$ due to the velocity components $u(x, y, t)$ and $v(x, y, t)$ is for all time the given constant $W$

$$
\int_{q_{1}} u(x, y, t) d x+v(x, y, t) d y=\int_{R} h(x, y) \zeta(x, y, t) d x d y-f(t) \int_{q_{1}} \frac{\partial h}{\partial \nu} d s=W .
$$

This verifies the statement $(j)$, and the other conclusions of Theorem $I$ are 
verified as before. To infer the conclusion (d) we know that at time $t=0$ the velocity components $u(x, y, 0), v(x, y, 0)$ and the given velocity components $u(x, y), v(x, y)$ have the same vortex density $\zeta(x, y, 0)=\zeta(x, y)$, the same circulation, $W$, around the inner boundary, and divergence zero.

Using the transformation of variables that was employed in proving the existence of $\partial \phi / \partial t$ it can be shown that $d f / d t$ exists,

$$
\frac{d f(t)}{d t}=\frac{1}{\lambda} \int_{R}\left\{u(x, y, t) \frac{\partial h(x, y)}{\partial x}+v(x, y, t) \frac{\partial h(x, y)}{\partial y}\right\} \zeta(x, y, t) d x d y .
$$

Then $\partial \phi / \partial t$ exists and is continuous,

$$
\frac{\partial \phi(x, y, t)}{\partial t}=-\frac{1}{2 \pi} \int_{R} \zeta\left(x^{\prime}, y^{\prime}, t\right)\left\{u \frac{\partial G}{\partial x^{\prime}}+v \frac{\partial G}{\partial y^{\prime}}\right\} d x^{\prime} d y^{\prime}+h(x, y) \frac{d f}{d t} .
$$

Then from potential theory we know that the first order partial derivatives of $\partial \phi / \partial t$ with respect to $x$ and $y$ exist and are continuous. By continuity the order of differentiation is immaterial so $\partial u(x, y, t) / \partial t$ and $\partial v(x, y, t) / \partial t$ exist and are continuous in $x, y, t$.

To construct the pressure, let $C$ be a closed Jordan curve in $R$ with continuously turning tangent and moving with the fluid. If $C$ encloses the inner boundary, $q_{1}$, of $R$ we have

$$
\int_{C} u d x+v d y=\int_{q_{1}} u d x+v d y-\int_{R_{C}} \zeta(x, y, t) d x d y,
$$

and it follows from the constancy of the circulation around $q_{1}$ and the constancy of $\int_{R_{C}} \zeta(x, y, t) d x d y$ that the circulation around $C$ is a constant independent of the time. Thus if $C$ is any closed curve moving with the fluid, whether or not $C$ encloses the inner boundary of $R$, the circulation around $C$ is a constant independent of the time. Then it follows that

$$
\int_{C}\left\{\left(u \frac{\partial u}{\partial x}+v \frac{\partial u}{\partial y}+\frac{\partial u}{\partial t}\right) d x+\left(u \frac{\partial v}{\partial x}+v \frac{\partial v}{\partial y}+\frac{\partial v}{\partial t}\right) d y\right\}=0
$$

and a single valued pressure can be constructed satisfying the Euler dynamical equations.

Purdue UnIVERSITY

LAFAYETTE, IND. 